Euromath Bullewin

Vol 2, No. 1,1996

\title{
Securing the Net - the Fruits of Incompetence
}

\author{
Arjen K Lenstra \\ Roort MCC-1C317B, Bellore, 455 south Street \\ Mortistown, NJ 07960-6438 \\ U. S. A \\ lenstrafobellore.com
}

\begin{abstract}
This note reviews the most popular mathematical primitives that are used in current attempts to build secure networks.
\end{abstract}

\section{Introduction}

Corporations worldwide suddenly regard the Internet; once the almost exchusive play zround of the global comminity of computer nerds, as an immense business opporturity. Until recently phraes such as "consult our homepage at http://ww. di gicrime . com" made sense to only a fow; now they belong to our everyday vocabulary. This is all part of the explosive growth of what has been called the Global Information Infrastructure. It is a developnent that cannot be stopped and probably should be welcomed.

Nevertheless, corporations are beginning to sce that venturing out on the Internet way expose them to enormous risks. The purpose of "puttirg your homepage on the web" is to increase visibility and to draw attention. Unfortunately the audience includes not only potential customess but also virtually all hackers world wide. At least some of them will, intentionally or not, cause trouble.

Sclutions to the resulting secirity problems are not hard to find on the net, since mainy software vendors now advertise "secure" versions of their products. This makes using the net really risky, because users might mistakenly believe they are well protected. The widely publishized and rather frecunent news stories about network break-ins and imperfections ir security software should dispel such illusions. It seens that our competence to secure the net cannot keep up with our dosire to use it

Despite the confusing array of security solutions, there are only a few mathematical primitives on which they are based. Even in fauty sccurity products, the soundness of the underlying mathematics is hardly ever in question it is the way it is used that causes the vilnerabilities. In this note I discuss the mathernatical primitives-zot the mary slippery ways in which they are employed. I concentate on the primitives themselves and the assumption of their soundness and will show that this is one of the most inportant reasons that computational number theory has become so fashionable, even at industrial labs. This popularity, based entirely on our incompetence at efficiently solving a few basic number theoretic problems, is hardly something of which to be proud. Furists who object to the vilgatiastion of number theory should find comfort in the prospect thet as soon as efficient solutions have been found. 
Arjen K. Leastra

number theory will again be the immaculately impractical Quen of Mathematics-its status before "sccurity applicetions" came along.

This note is w:itten for an undergraduate mathematics audience that is not famillar with the mathematical notions involved in many popular security products. In Section 2 I sketch a possible security application on the Internet. Of course ruch more is involved in practice than I and able to mention here, but I show some of the basic concepts and set the stage for the mathematical primitives that are presented in Sections 3 and 4 No attempts are mede to formalize notions such as "infeasible", "hard" or "efficient". For further background refer to [2] (Section 2) and [1] (Sections 3 and 4).

\section{Background}

The following naive scensnio, though grossly oversimplified, shows some of the key issues of communication security. Suppose that two parties who have never met want to exchange confidential information over sorre untrusted but reliable network. "Untrusted" here means that all messages are accessible to eavesdroppers; "Ieliable" means that no bits are dropped or changed. The Internet is a reasonable example of such a network.

If the two parties share a random string $s$ of secret bits that is as long as the messagem, then the problem can easil $y$ be solved send the biturse exclisive or $s \oplus \mathrm{m}$ of $s$ and $\mathrm{m}$. Sinces is rancom, $s \oplus \mathrm{m}$ leaks no information. Futhormore, it is easy to derive $m$ from $s \oplus m$ because $m=s \oplus(s \oplus m)$. This would solve the problem, except that we cannot assume that any two parties have s secret string of random bits in common. A further disadvantage is that, using this simple approsch, each $s$ can be used only once (since $s \oplus m_{1}$ and $s \oplus m_{2}$ rcved infomation about $m_{1} \oplus m_{2}=\left(s \oplus m_{1}\right) \oplus\left(s \oplus m_{2}\right)$ ).

The latter problen can be overcome by using, for instance, DES-the U.S. government's Data Encryption Standard. DES is an example of a block copher. It can be used to construct a function $f$ such that it is sufficiently hard to derive $m$ from $f(s, m)$ (for any number of messages $m$ of any length), such that $m=f(s, f(s, m))$, and such that $f$ can very efficiently be computed. Here $s$ is a 56 -bit string, or a 168-bit string if higher security is needed (triple-DES); this string is referred to as the key. This, if both parties had a common key $s$ that was unknown to any other party, any message $m$ could be encrypted as $f(s, m)$, sent to the other party using an untrusted channel, and decrypted as $n=f(s, f(s, m))$. This can be repeated back and forth, for any reasonable number of messages.

A descrivtion ofDES is beyond the scope of this note; it does a lot of seemiggly arbitrary bit-fidding that aims to, anong other things, confuse and diffuse the bits of the ley s and the message m. There are many other ciphers that can be used to construct functions that have properties similar to $f$. For orr puposes the problem that remains to be considcred is how the two parties perform the key exchange for a reiatively short key (of, say, 56 bits), in such a way that the key that is exchanged remains hidden to an eavesdropper.

Note that the sinple approech where the (trusted) provider of the comminicetion services assigns a unique randon key to each pair of possible parties is not fessible: each party would need an enonnous data base ofkeys, which vould not only be hard to keep updated (for new subscribers) but would also have to be safegtlarded very carefully. An elegant solution to the problom of key exchange is given in Section 4. It only requires a small amount of public information that is accessible to the entire newwork. While using it; however, all parties involved need to sign all their messages.

This requires digital signatures to convince each of the communicating partics that the messages they receive come from the party they intend to commumicate with. This can be realized using public key cryptography, as explained in the next sections. In public key cryptography all parties huve a secret key and a comesponding public key. In signature applications the secret kcy is used by its owner to generate a signature; the corresponding public key can be used by anyone to check the validity of the signature. 
Thus, all secret keys are kept hidden by their owners, but all parties have access to each other's pubilc keys, just as telephone numbers are (mostly) public information. Alternatively, parties that wish to communicate can exchange their public keys first; this in turn leads to the problom of authenticating public keys and related issues, which can abo all be solved using public key cryptography (and which may, in certain circumstarces, require a trusted third party). In practice many othor problems have to be addressed as woll. The pupose of our simpleminded example is only to introduce the basic principles as a background for the ruathematical primitives that are presented below.

A cyptographic technique that is often used for digital signatures in conjunction with public key ayptography is hashing. A hast function $h$, when applied to a message $m$ of arbitrary length, results in a fixed length hash $h(m)$ of $m$; for MD5 (Message Digest) the resulting length is $128 \mathrm{bits}$, for SHS (the U.S. government's 'Secure Hash Standard') it isl60 bits. For a good bash functior it should be infeasible to compute an $m$ such that $h(m)$ is equal to any prescribed value. Also, it should be infeasible to find different $m_{1}$ and $m_{2}$ such that $h\left(m_{1}\right)=h\left(m_{3}\right)$. Iike DES, the currently popular hash functions are based on very efficient seemingly rendom bit manipulations, and not on clear-cut mathematical ideas as most public key cryptosystems are (even though those ideas might turn out to be incorrect). It is an open problem how to design a very effecent hash function that is provably as hard to break as one of the public key cryptosystems described below. It is also becoming an urgent problem: on May 2, 1996. Hans Dobbertin of the German Information Security Agency, who was responsible for breaking MD4 in 1995. announced a new cryptanalysis of MD5 that 'might be reason exough to substitute MDS in future applicatious'. The life expectancy of SHS is uncetain, since its design is very similar to that of MD5.

\section{Factoring}

Faxtoting a composite integer $n$ means finding integers $p$ and $q$, both $>1$, such that $n=p-q$. This is, in its generality, believed to be e hard problem, even though there is no firm mathematical ground on which this assumption can be based the only evidence is our failure to find an effient factoring method.

The supposed difficulty of factoring is crucial for the security of the RSA public key cryptosystern, which was invented in 1977 by Rivest, Shamir, and Adleman. Each nser of RSA has its own modulus $n=p \cdot q$. where $p$ and $q$ are two large primes, and two irtegers $e$ and $d$ such that $e-d \equiv 1 \bmod (p-1)(q-1)$. The values $n$ and $e$ are made public as that user's public key, but $d$ is kept secret (along with $p$ and $q$ ) and is the uscr's secret key. Since large primes can efficiently be generated, and because $d$ can quickly be found using the extended Euclidean algonthm giver $e, p$, and $q$ (for propcrly chosen $e$ ), suck $r$, and $d$ can easily be found for each user of RSA.

To send a message $m \in Z / n Z$ to the owner of public key $(n, e)$, one computes $E(m)=m^{2}$ and transmits the encrypted massage $E(m)$. The ownor of $(n, e)$, upon receipt of $E(m)$, retrieves the tunencrypted message $m$ by computing $E(m)^{d}=m$ (cf. Fermat's little theorem). Both the encryption and the dexyption can efficiently be done using the 'repeated square and rultiply' method. This is, however, considerably slower than, for instance. DES. RSA car also be useri to sign a message $m$ as $S(\pi)=m^{d}$; the validity of the signature can be checked by verifying that $S(m)^{e}=\pi v$

It bas not been proved that factoring $\pi$ is necessary to break RSA (i.e., to decrypt $E(m) \mathrm{knowizg}$ $n, e$, and $E(m)$ but without knowing the proper $d)$, but it is obviousiy sufficient. There exist several variations of RSA that replane the multiplicative group $(\mathbf{Z} / \pi \mathbf{Z})^{*}$ by some other group that depends on a composite roduius, and in which the operations are carricd out. Nonte of them, however, seems to have any substantial advantages over RSA (despite clairns to the contrary), and all of them can be broken by factoring the modulus. 
Arjen K. Lenstra

Since the invention of RSA considerable progress has been made towards more efficient factoring methods. Trial division and Pollard's rho method (1975) find the smellest prime factor $p$ of $\pi$ in exponential time: $O(p)$ and $O(\sqrt{p})$, respectively. The elliptic curve method (ecm, 1985) can be expected, on loose heuristio grounds, to take time $(\log \pi)^{2} \exp ((1+o(1)) \sqrt{2 \log p \log \log p)}$, for $p \rightarrow \infty$. Note that this is subexponential in $p$. These are examples of special purpose factoring methods, since their nu time deperds on the factors to be found. The largest factor found by any of these methocs (ecm) so far has 47 decimal digits. None of them is a threat to RSA, if the primes in the modulus have at least, say, 75 decimal digits.

The run time of general purpose factoring methods depencis solely on the size of the aumber to be factored. The most important ones are the Continued Fraction method (CFRAC, 1970), Quadratic Sieve (QS, 1981), and the Number Field Sieve (NFS, 1989). The first two have herristic expected run time $\exp (1+o(1)) \sqrt{\log n \log \log n})$ for $n \rightarrow \infty$, though $Q S$ is in practice much to be preferred to CFRAC They share this rin time with many other factoring algorithms, among others the worst case $p \approx \sqrt{n}$ of ectr. NFS was the first algorithm to substantially improve upon this time, with hewristic expected rum time $\exp \left((c+o(1))(\log n)^{1 / 3}(\log \log n)^{2 / 3}\right)$ for $n \rightarrow \infty$ and csome constant between $\approx 1.53$ (for 'nice' numbars like $2^{2^{4}}+1$ ) and $\approx 1.92$ (for general numbers). NFS is currently consicered to be more efficient than QS for numbers that have nore than, say, 110 decinal digits.

The largest number factored with QS, in 1994, is the famous 129-digit 'RSA-challenge' that appeared in the August 1977 issue of Scientific American. Rivest estimated, in 1977, that this factorization would requirc 40 quadrillion years. With $Q S$ it took 8 months, using the idle cycles of computers world wide. The total run time of this factoring effor has been estimated as 5000 mips years, (ie., 5000 years on a VAX 780). The largest number factored with NFS, in 1996, is a 130-digit RSA-modulus; with total ran time estimated as 550 mips years. Using this figure and the asymptotic run time of NES (omitting the $o(1)$ for convenience), one can get an inpression of the effort required to factor 512-bit (155-digit) RSA moduli, nod conclude that such moduli are on the verge of being breakable. With a moderate amount of progress in factoring, 768 -bit keys (a size that is becoming thore popular lately) could become vulnerable as well. A polynomial-time factoring algonithm wonld mrost likely render RSA useless.

\section{Discrete logarithms}

The most common discrete logarithm problem is the following. Given some prime $p$, a generator $g$ of $(\mathbf{Z} / p \mathbf{Z})^{*}$, and $y \in(\mathbf{Z} / p \mathbf{Z})^{*}$, find $x \in\{0,1, \ldots, p-1\}$ such that $g^{x}=y$. Like factoring, this is in its generality believed to oe a hard problem, and, again lise factoring, the orly evidence that it is hard is that we have not yet been able to solve it efficientily.

The supposed diffculty of discrete logarthns is the basis for the security of the Diffe-Hellman hey exchange protocol (1976). A prime $p$ and generator $q$ ere publicly known. Party $A$ picks a random $a \in\{0,1, \ldots, p-1\}$, computes $g^{a} \in(\mathbf{Z} / p Z)$ and sends it to party $B$. Party $B$ picks a random $b \in\{0,1, \ldots, p-1\}$, computes $g \in(\mathbf{Z} / p Z)^{*}$ and sends it to $A$. Both parties can now compute the common key $g^{a b}=g^{b t} \in(\mathbf{Z} / p \mathbf{Z})^{*}$. As mentioned earlier, this key exchange protocol should be used with care, since otherwise it is susceptible to as mon in the mid de attack.

It has not been proved that computing discrcte logarithms is necussary to break the Diffe-Heliman protocol (i.e. to compute $g^{a b}$ given $p, g, g^{a}$, and $g^{b}$ ), but it is obviously sufficient. Many other public key cryptosysterns have becn proposed that car be broken if discrete logarithms can be cornputed efficiently. 


\section{Secuing the Net - the Eruits of Incompetence}

However, mike factoring based systems which are more or less equally hard to brcak, here the situation is a bit nore complicated.

It does not seern to be possible to reduce fsctoring to discrete logarithms, or vice versa Nevertheless, there is a strong similarity between the solution methods for the two problems: with a few exceptions (such as ecro), the ideas behind nost factoring algorithms can be used to solve discrete logarithms as well. Examples are linear search for $x$ and Pollard tho, which find $x$ is time $O(x)$ and $O(\sqrt{x})$ operations in $(\mathrm{Z} / p \mathrm{Z})^{*}$, respectively. The 'Gaussiar integers' method finds $z$ in time $\exp ((1+o(1)) \sqrt{\log p \log \log p})$, for $p \rightarrow \infty$, and is based on ideas similar to the ones that lod to QS. And then there is a Nimber Field Sieve based method that finds $z$ in time

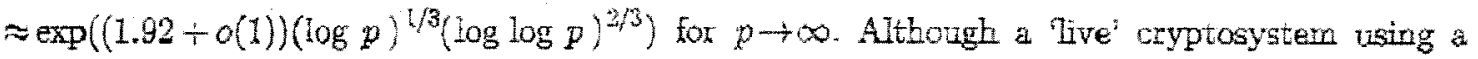
60-digit prime was broken in 1991, practical experience with discrete logarithm algorithms is limited. Efforts are underway to change this situation.

An important distinction betwen the exponential and subexponential time discrete logkinthm methods is that in the former the group $(\mathbf{Z} / \mathbf{Z Z})^{*}$ can be replaced by any group, but in the latter; artituetic properties of the set $\{1,2, \ldots, p-1\}$ (which is used to represent $(Z / p Z)^{*}$ ) are crucial This has several imteresting consequences, of which I mention a few.

If $g$ generates only a subgroup of order $q<p-1$ of $(Z / p Z)^{*}$, and $y \in<g>$, then $x$ can still be found in $O(\sqrt{x}) \leq O(\sqrt{q})$ operations in $(\mathrm{Z} / \mathrm{ZZ})^{*}$ (using Pollard's tho method, or using Shanks: 'baby-step-giant-step' method), or in time subexponential in $p$ using any of the subexponentid methods. But a method that runs in time subexponential in $q$ is not knowm. If $g$ generates the group of points of some elliptic curve modulo $p$, then $x$ can again be found in $O(\sqrt{x})$ operations in the elliptic curve group. But no method is known that rums in time subexponential in the order of $g$ or even in $p$. If, on the other hand, $g$ generates $\left(F_{m^{m}}\right)$ or a subgroup thereof, for some fixed integer $m>1$, then $x$ car be found in time subexponential in $p^{m}$. The latter is a consequence of the fact that the relevant arithmetic properties of the set of polynomials modulo $p$ of degree $<m$ is similar to those of the set $\left\{1,2, \ldots, p^{m}-1\right\}$.

This anparent leck of discrete logarithm algoritims thet run in time suvexponential in the order of $a$ subgroup or of an entirely different group that cannot be represented in the way the subexponential algorithms require, is cxploted in the design of screral public key systems. In DSS (the U.S. goverument's Digital Signature Standeard) an order $q$ subgroup of $(\mathbf{Z} / p \mathbf{Z})^{*}$ is used to traprove the speed of the cryptosystem, while st the same time reducing the sige of the resulting signatures, without, hopefully, compromising the security: breaking it requires time esther $O(\sqrt{q})$ or $\approx \exp \left((1.92+o(1))(\log p)^{1 / 3}(\log \log p)^{2 / 3}\right)$, both of which are supposedly infearible for the specified sizes $\left[\log _{2} q\right]=159$ and $\left[\log _{2} p\right] \geq 511$ (the Russian variant of DSS requires $\left.\left[\log _{2} q\right]=239\right)$. Elliptic curve based cryptosystems achieve the same objectives simply by choosing $p$ swall, but large enough to make $O(\sqrt{p})$ attacks infeasible.

Since $\left[\log _{2} q\right]$ is fixed at 159 and the increase of processor speed has not levelel off yet, it is conceivable that a moderste amount of progress in exponentinl time discrete logarithm algorithms could meke DSS vulnerable within the foreseeable futurc Also, some specialists find it too early to give up hope for better than exponential time attacks on ellptic curve based systerns. 
Arjen Ǩ. Lenstra

\section{Conclusion}

We have seen that the factoring and discrete Iogarithm problems are remarkably similar: both are easy to formulate, believed to be hard purely based on our lack of success soiving them efficiently, suitable for the design of public key cryptosystems, and, most remarkably, they seern to be susceptible to very similar solution methods. The last point is quite worrisome: even though not ell factoring methods a an be turned into discrete logarithm methods (ecm is the most notable exception), Enost can. Thus, it is conceivable that a newly invented factoring method that wipes out all factoring besed cryptosystems, would have the seme effect on discrete Iogerithm based cryptosystems. Obviously, there is a strong need for diversification in the design of public key cryptosystems.

These isanes, though crucis lor the design of secure networks, are actually the least of ou current worries. It is not wnikely that instead of enjoying the fruits of our number theoretic incompetence we will soon be harvesting the rotten fruits of our incompetence at properly implementing or use the much needed security measures. As soon as we leave the realm of mathematics, security considerations become much more confused and complicated: human factors, compatibility issues, trust chanagcment, key management, key escrow, export restrictions, to mention only a few; are crucial isstes that have an enormous potential to be exploited by a worldwide arny of hackers that cannot necessarily distinguisin a prime from a composite. This is not to say that security on the net cannot be axtieved, but the subject requires study of much more than the mrathernatical issues alone.

\section{References}

[1] A. K. Lenstrand H. W. Lenstra, Jr. Handbook of theoretical computer science. Volume A, Algorithms and complexity. (J. var Leenwer, ed.). Algorithms in number theory. Chapter 12. Elsevier. Amsterdam. 1990.

[2] D. R. Stinson. Cryptography, theory and practice CRC Press. Boca Raton. London, - Tokyo 1995. 